\title{
Tratamiento quirúrgico de la isquemia mesentérica crónica: Caso clínico
}

\author{
Juan Marín P, Luis Manríquez C, Helmuth Schweizer H, \\ Juan Tellez M, Gonzalo Rossel D.
}

Surgical treatment of chronic mesenteric ischemia: Report of one case

\begin{abstract}
Although the classic therapy for chronic mesenteric ischemia is surgical revascularization, endovascular therapy is a new therapeutic option. We report a 55 year-old female, with a 2 years history of post prandial abdominal pain, diarrhoea, and weight loss, with occlusion of both mesenteric arteries and critical stenosis of the celiac artery. The initial treatment consisted in angioplasty and celiac artery stent placement in two occasions, with a brief symptomatic relief. Finally, a visceral artery bypass was performed, with good post operative outcome and complete symptomatic resolution at one year follow up. In our opinion endovascular therapy is a good therapeutic option for chronic mesenteric ischemia in high surgical risk patients, specially when dealing with stenotic injuries. It may also be a complement for patients who need to recover their nutritional status prior to revascularization surgery. On the other hand, due to the long term patency and symptomatic relief, surgical treatment is a good option in low risk patients (Rev Méd Chile 2007; 135: 92-97).
\end{abstract}

(Key words: Embolism and thrombosis; Ischemia; Mesenteric arteries)

Recibido el 11 de agosto, 2005. Aceptado el 4 de mayo, 2006.

Servicio de Cirugía y Radiología, Hospital Militar de Santiago.

L a isquemia intestinal crónica es un cuadro poco frecuente, que está asociado a una alta morbilidad y mortalidad, principalmente por su complicación que es el infarto intestinal masivo ${ }^{1}$. Su principal etiología es la enfermedad ateromatosa oclusiva de los troncos viscerales. Tradicionalmente, el tratamiento ha sido la revascularización quirúrgica, sin embargo, en la actualidad ha surgido la terapia endovascular como alternativa ${ }^{2}$.

En nuestro medio, sólo hay dos publicaciones respecto al tratamiento de esta enfermedad ${ }^{3,4}$,

Correspondencia a: Juan Marín P. La Araucana 728, La Reina, Santiago. Fax: 2368726. E mail: pajua@entelchile.net motivo por el cual, el objetivo de esta publicación es comunicar un caso clínico de isquemia mesentérica crónica tratado primariamente con terapia endovascular en dos ocasiones, siendo insuficiente, con recurrencia precoz de la sintomatología, por lo que se sometió a tratamiento de revascularización de los troncos viscerales, a través de la cirugía vascular convencional.

\section{CASO CLÍNICO}

Paciente mujer de 55 años, con antecedentes de tabaquismo crónico, hipertensión arterial modera- 
da y dislipidemia en tratamiento, cuyo cuadro comenzó en el año 2002, caracterizado por dolor abdominal tipo cólico postprandial precoz, acompañado de diarrea frecuente, alrededor de 10 episodios diarios y vómitos ocasionales, además de baja de peso de alrededor de 15 kilos, siendo su peso habitual de $55 \mathrm{~kg}$, llegando a un índice de masa corporal de 17,3. Estudiada en el contexto de diarrea crónica y búsqueda de enfermedad inflamatoria intestinal, se sometió a endoscopia alta y baja, además de enteroscopia, tránsito intestinal y estudio de mala absorción, lo que sólo concluyó gastritis y colitis inespecífica, no respondiendo a tratamiento habitual, esto es, régimen alimenticio, antiespasmódicos y medicamentos antiulcerosos. En enero de 2004 se le efectuó un ecodoppler de arterias viscerales, demostrándose una velocidad de $400 \mathrm{~cm} / \mathrm{seg}$ en tronco celíaco, compatible con estenosis de $90 \%$, oclusión de la arteria mesentérica superior en su origen y llene por colaterales, además de oclusión de la arteria mesentérica inferior. Una angiotac de abdomen confirmó estos hallazgos (Figura 1).

Dada las condiciones de desnutrición importante de la paciente, se le consideró de alto riesgo quirúrgico, por lo cual, en marzo de ese año, se le practicó estudio angiográfico y se le instaló un stent balón expandible de $5 \mathrm{~mm}$ por $17 \mathrm{~mm}$ a nivel de tronco celíaco (Figura 2), con lo cual desapareció su sintomatología con recuperación de peso hasta 50 kilos. Sin embargo, a los dos meses reapareció el dolor abdominal y la diarrea, por lo cual se le controló con ecodoppler abdominal que reveló velocidades de $400 \mathrm{~cm} / \mathrm{seg}$ a nivel de tronco celíaco, compatible con estenosis significativa. Se efectuó nueva angiografía, donde se pesquisó oclusión de stent de tronco celíaco, procediéndose a instalar un segundo stent balón expandible en la porción proximal del tronco celíaco. Permaneció asintomática hasta noviembre, en que nuevamente comenzó con dolor, diarrea y vómitos. Un tercer ecodoppler de arterias viscerales demostró velocidad de $417 \mathrm{~cm} / \mathrm{seg}$ en tronco celíaco, por lo cual se sometió a un tercer estudio angiográfico que mostró stent de tronco celíaco permeable, pero con estenosis significativa (Figura 3).

Después del último estudio angiográfico, hecho por vía femoral derecha, utilizando siempre esta misma vía de acceso para los otros estudios angiográficos, la paciente experimentó dolor tipo

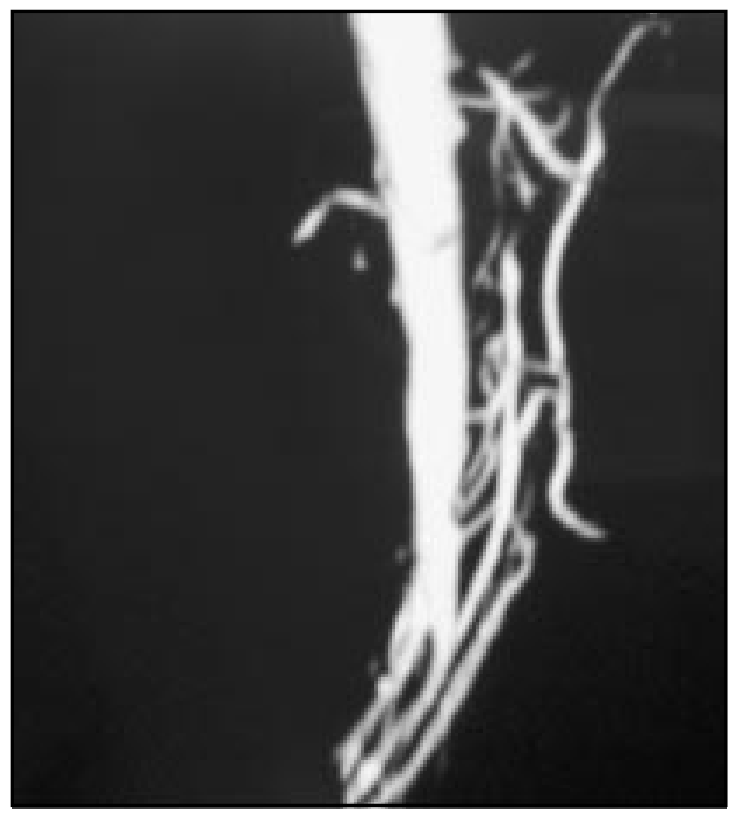

Figura 1. Angiotac de abdomen que revela oclusión de ambas arterias mesentéricas y estenosis crítica de tronco celíaco.

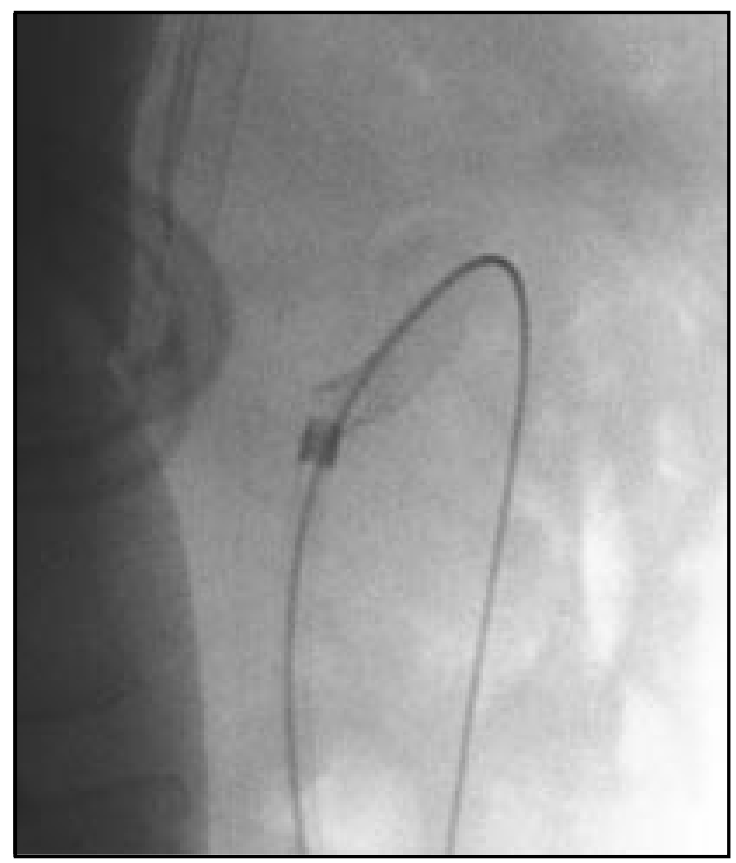

Figura 2. Angioplastía y stent de tronco celíaco. 


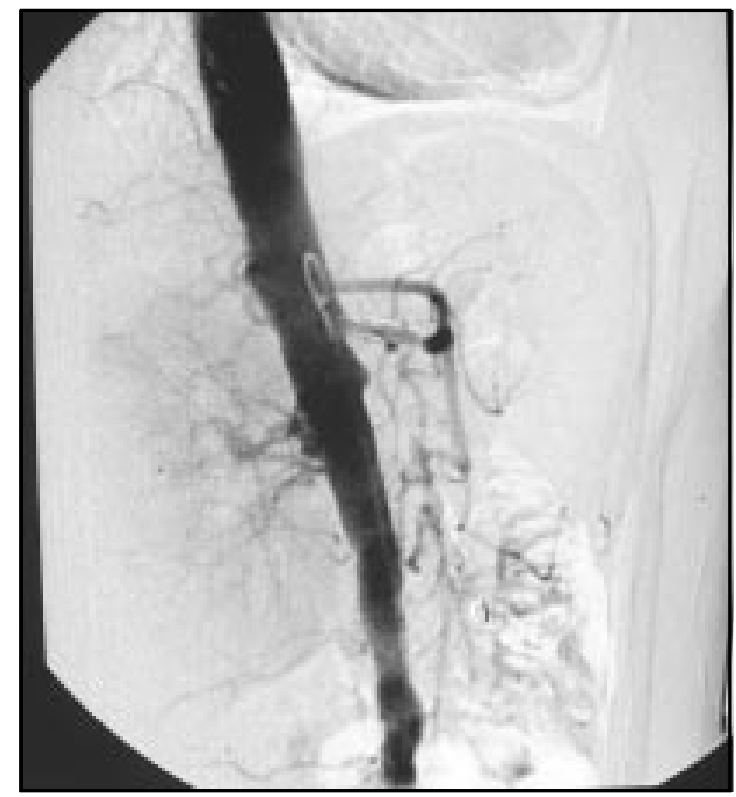

Figura 3. Angiografía que revela segundo stent de tronco celíaco permeable, pero con estenosis significativa.

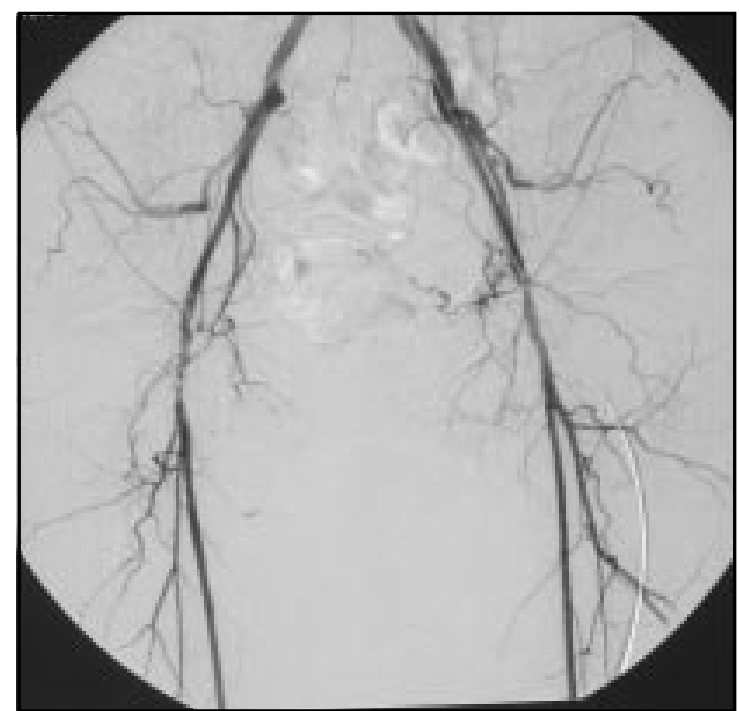

Figura 4. Estenosis segmentaria severa de arteria femoral común derecha, posangiografías repetidas por la misma vía.

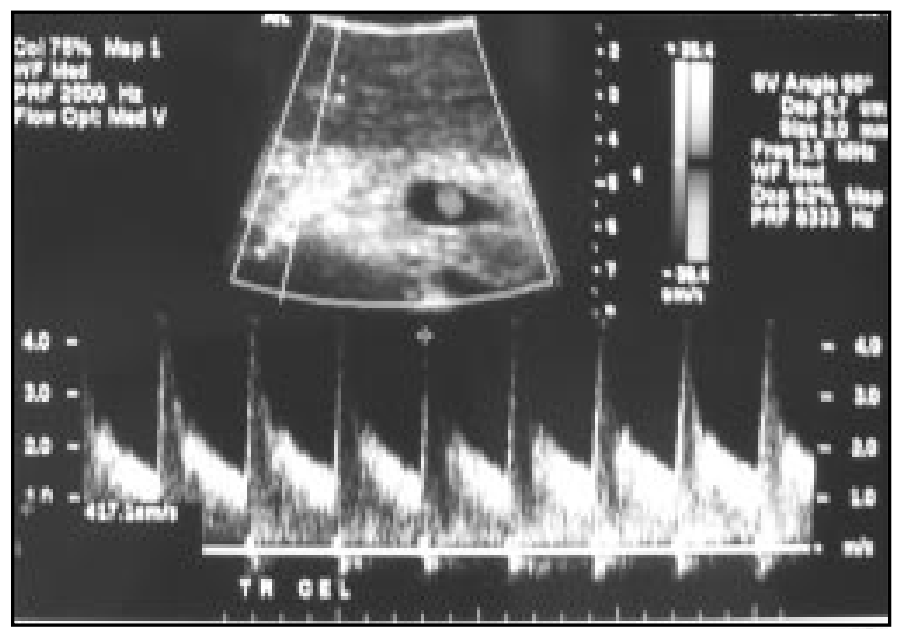

Figura 5. Ecodoppler que revela estenosis significativa de tronco celíaco, con velocidad de $417 \mathrm{~cm} / \mathrm{seg}$.

claudicación que se hizo invalidante, por lo que en diciembre, nuevamente reingresó por isquemia severa de esa extremidad. Nuevo estudio angiográfico reveló estenosis segmentaria de arteria femoral y trombosis de arteria tibial anterior, (Figura 4) cuadro que se resolvió con angioplastia con balón, mejorando la isquemia de su extremidad.
Debido a la persistencia de sus molestias y baja de peso de alrededor de 5 kilos, consultó en enero de 2005 en nuestro hospital, esta vez con el equipo de cirugía vascular en donde se le practicó un nuevo ecodoppler de arterias viscerales que demostró velocidad de $470 \mathrm{~cm} / \mathrm{seg}$ a nivel de tronco celíaco, compatible con estenosis crítica (Figura 5). 
Se le propuso una revasculanización quinúrgica. Se evaluó desde el punto de vista cardiológico con electrocardiograma y ecocardiograma que fueron normales. Entre los exámenes de sangre sólo destacaba anemia con hematocrito de $20 \%$ secundario a su estado nutricional, con peso de 45 kilos. Finalmente, el 22 de febrero, se le practicó un puente aortomesentérico superior retrógrado con prótesis de PTFE anillado de $6 \mathrm{~mm}$ y un reimplante de la arteria mesenténica inferior en el origen de la prótesis (Figuras 6 y 7). La evolución posoperatoria fue sin complicaciones, con realimentación sin problemas, desaparición del dolor postprandial y la diarrea, además de recuperación de su peso. A la fecha de esta comunicación, al año de operada, un angiotac reveló permeabilidad de la revascularización (Figura 8).

\section{DisCUSIÓN}

La isquemia mesentérica crónica es un cuadro poco frecuente. Sin embargo, su principal complicación, el infarto intestinal masivo, resulta en una alta morbimortalidad. Su principal etiología es la enfermedad ateromatosa oclusiva de los tres principales troncos viscerales que irrigan el intestino, que con frecuencia afecta los orificios de estas arterias.

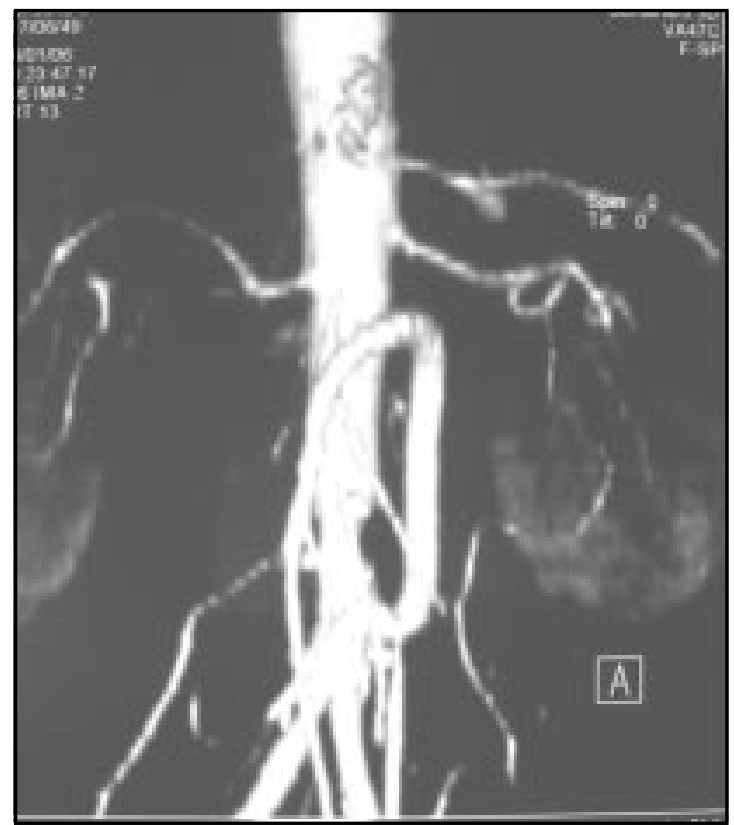

Figura 8. Control de puente a arterias mesentéricas que revela prótesis permeable (angiotac).

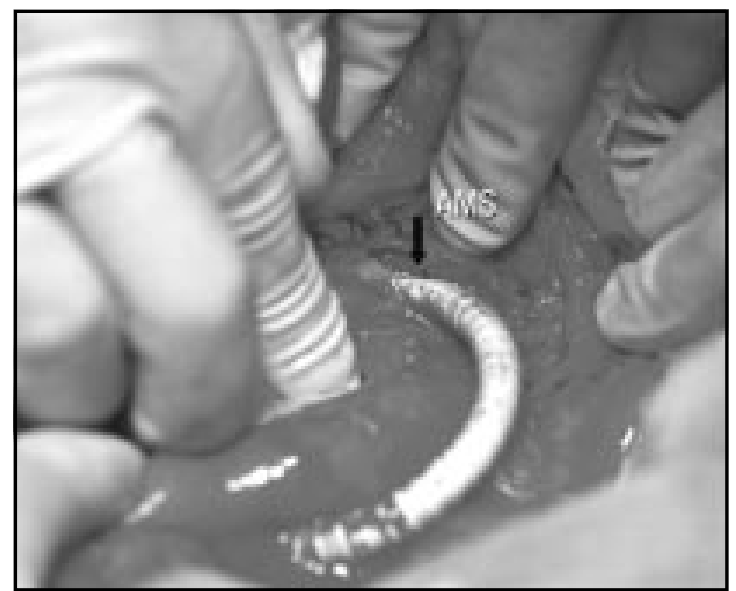

Figura 6. Puente de revascularización con prótesis de PTFE anillado desde aorta distal a arteria mesentérica superior (AMS: arteria mesentérica superior).

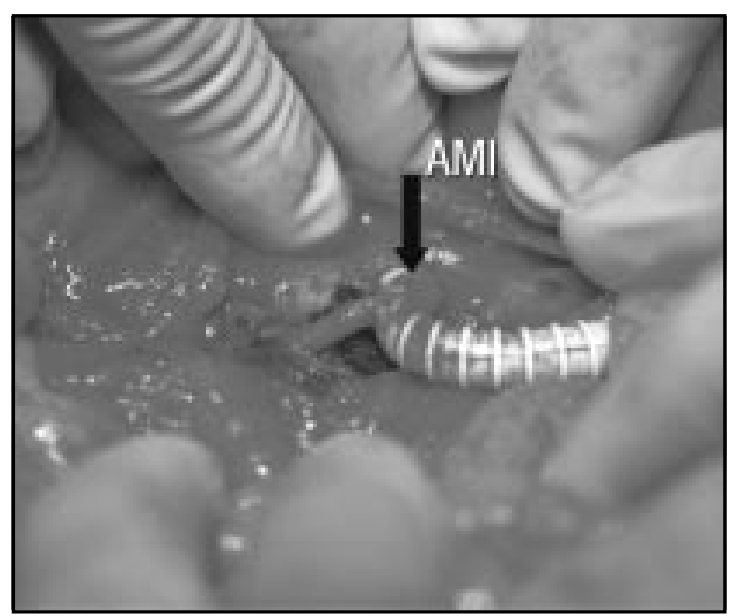

Figura 7. Reimplante a arteria mesentérica inferior en el inicio de la prótesis (AMI: arteria mesentérica inferior).

No hay exámenes de laboratorio específicos que permitan confirmar el diagnóstico. Casi siempre son pacientes que son estudiados por cuadro de diarrea crónica, dolor abdominal y baja de peso buscando una neoplasia digestiva, sin embargo, hoy en día se dispone del ecodoppler como método no invasivo que permite evaluar la presencia de estenosis u oclusión de los troncos viscerales. Este examen requiere de un profesional entrenado, por lo cual tiene el inconveniente de ser operador dependiente ${ }^{7}$. Si bien hoy en día también se cuenta con la tomografía computarizada y la angiorresonancia, la angiografía sigue 
siendo el examen de elección para su diagnóstico y para planear el tratamiento a seguir ${ }^{8}$.

Tradicionalmente, el tratamiento de esta enfermedad ha sido la revascularización quirúrgica de los troncos viscerales, entre los cuales están los puentes de revascularización como el método más usado. Pero también se han practicado endarterectomías y reimplantes de estos troncos.

Cada uno de estos métodos tiene ventajas y desventajas, debiendo ser aplicado de distintas maneras de acuerdo a la experiencia del cirujano, las condiciones locales del paciente y también si es una revascularización adicional, con una mortalidad que fluctúa entre $0 \%$ y $11 \%$, cuando son efectuadas en forma electiva, con morbilidad hasta $32 \%$, principalmente relacionadas con la avanzada edad, estado cardiovascular y en menor grado con el fracaso de la revascularización ${ }^{9,10}$.

Desde la década 1980-89 se ha propuesto la terapia endovascular para tratar esta enfermedad, la angioplastia con balón y stent ${ }^{11}$, técnica percutánea y mínimamente invasiva, con cifras de menor morbilidad y mortalidad en comparación a la cirugía tradicional ${ }^{12}$. Sin embargo, sus resultados, si bien, ofrecen buen éxito inicial, especialmente en relación al tratamiento de las estenosis de los troncos viscerales, a la fecha tienen un mayor porcentaje de reestenosis con mayor recurrencia de síntomas que la cirugía de revascularización ${ }^{13}$.

En el caso específico de esta paciente, es importante recalcar algunos aspectos en relación al tratamiento ofrecido:

a) Esta paciente se consideró de alto riesgo quirúrgico debido a su estado nutricional, en cir-

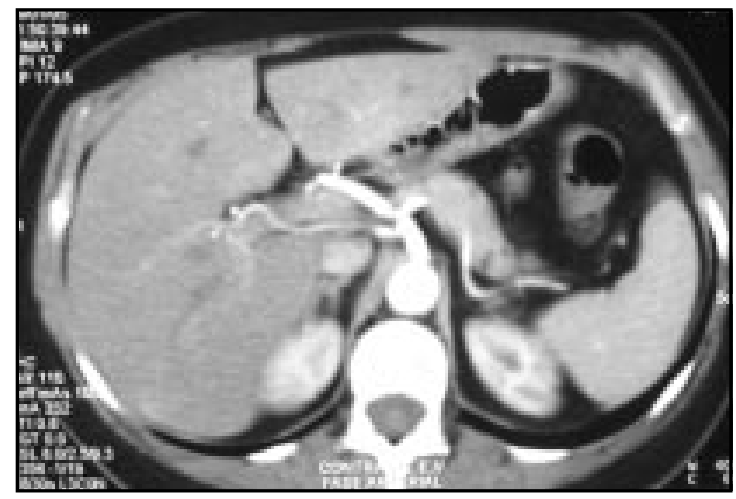

Figura 9. Angiotac de abdomen que revela estenosis de tronco celíaco en la parte distal de éste. cunstancias que esta enfermedad siempre se acompaña de baja de peso, por lo que se estima que esta condición no es contraindicación para la cirugía, ya que este problema se podría haber subsanado con alimentación parenteral, si las condiciones de desnutrición hubieran sido tan severas. b) Creemos que no fue una buena elección el tratamiento endovascular primario, ya que si hubiera fracasado esta terapia en un primer momento, habría significado un infarto intestinal masivo como complicación de una oclusión o una disección del tronco celíaco, puesto que era la única arteria que estaba imigando el intestino. Sin mencionar además, que la presentación de esta enfermedad a temprana edad de la paciente es una de las razones para haber optado por la cirugía abierta, dado sus mejores resultados a largo plazo. c) En relación a la instalación del segundo stent, en el angiotac da la impresión que existía una estenosis del tronco celíaco distal al stent, pero, además, en una angiografía parece ser más largo que el primero, que si bien no se dispone de la medida utilizada, parecería de una longitud de $34 \mathrm{~mm}$ (Figura 9 y 10), longitud bastante mayor que lo que mide el tronco celíaco, fenómeno que ayuda al desamollo de una hiperplasia miointimal que se da en la terapia endovascular y causa de reestenosis. d) Es muy importante el conocer la historia natural de la enfermedad, tener en cuenta la edad, las condiciones de comorbilidad y el riesgo quirúrgico. Por lo tanto, corresponde tomar una decisión entre gastroenterólogos, radiólogos y cardiólogos intervensionistas junto al cirujano vascular, para buscar la mejor

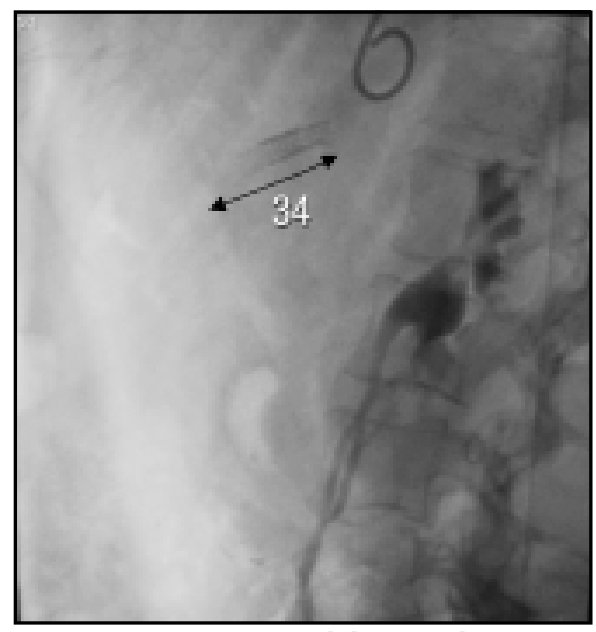

Figura 10. Imagen del segundo stent. 
opción terapéutica para cada paciente. e) Respecto al tratamiento quirúrgico, se optó por este tipo de revasculanización, ya que la aorta y las ilíacas de esta paciente tenían importante calcio en sus paredes, incluso en relación a la zona supracelíaca, por lo cual, se consideró no prudente efectuar un puente anterógrado hacia el tronco celíaco y la arteria mesentérica superior. Sólo había una pequeña zona de la aorta distal apta para intentar un puente de revascularización. f) En relación al tratamiento ideado para tratar la estenosis segmentaria severa de la arteria femoral común derecha, fruto de angiografías reiteradas y las dos terapias de angioplastia y stent, mediante una angioplastia con balón, sabemos que los resultados de esta terapia son malos en cuanto a la recurrencia a corto plazo de esta estenosis y en este segmento arterial específico y que en el caso de esta paciente, además de ser joven y sin contraindicación operatoria, habría sido mejor una terapia vía abierta convencional, aprovechando además de permeabilizar la arteria tibial anterior. Prueba de esto es que a 10 meses de la angioplastia, la paciente reinició la claudicación de su extremidad, que llegó a ser invalidante, por lo que debió efectuarse una angioplastia con parche del segmento iliofemoral, a

\section{REFERENCIAS}

1. FrY WJ, KRAFT RO. Visceral angina. Sur Gynecol Obstet 1963; 117: 417-24.

2. Kasirayan K, O'Hara PJ, Gray BH, Hertzer NR, Clair DG, Grenberg RK et al. Chronic mesenteric ischemia: open surgery versus percutaneous angioplasty and stenting. J Vasc Surg 2001; 33: 63-7.

3. VaLdÉs F, KRÄMER A, GuZMan S. Insuficiencia vascular mesentérica por enfermedad oclusiva crónica. Experiencia en el manejo quirúrgico de 9 casos. Rev Méd Chile 1989; 117: 653-62.

4. Duclos J, Urouieta M, Opazo M. Angina mesentérica: causa infrecuente de dolor crónico en paciente en hemodiálisis. Rev Méd Chile 2002; 130: 1155-8.

5. TAy LOR L, Moneta G. Intestinal Ischemia. Ann Vasc Surg 1991; 5: 403-6.

6. Baxter B, Pearce WH. Diagnosis and surgical management of chronic mesenteric ischemia. Surg Intervent Ther 1994; 795-802.

7. Moneta GL, Yeager RA, Porter JM. Dupplex ultrasound criteria for the diagnosis of splanchnic artery stenosis or occlusion. J Vasc Surg 1991; 14: 511-20. través de una cirugía abierta. g) Sin embargo, en este específico caso, la terapia endovascular, de alguna forma, ayudó a operar a esta paciente en una mejor condición nutricional que cuando fue sometida a la primera intervención, ya que había recuperado un mejor peso.

Finalmente, creemos que la terapia endovascular es una alternativa interesante en el tratamiento de la isquemia mesentérica crónica, de preferencia frente a lesiones estenóticas y focales, especialmente en el paciente de alto riesgo quirúrgico, incapaz de tolerar una revascularización quirúrgica tradicional. Incluso, esta terapia puede ser complementaria a la espera de recuperar un mejor estado nutricional antes de la cirugía definitiva. Sin embargo, es indispensable que esta patología sea discutida en conjunto entre el cirujano vascular y otros profesionales intervensionistas, ya que a la fecha, dado que las publicaciones son más bien casuísticas de pocos pacientes, todavía no está claro el rol en cuanto a cuál terapia sea la mejor para esta enfermedad, debiendo confrontar la baja morbimortalidad de la terapia endovascular con los buenos resultados a largo plazo y libre de reintervención de la cirugía abierta.

8. Crawford ES, Morris GC, Myhre HO et al. Celiac axis, superior mesenteric artery, and inferior mesenteric artery occlusion; surgical considerations. Surgery 1977; 82: 856-66.

9. Jiménez JG, Huber TS, Ozaki CK, Flynn TC, Bercel SA, LeE WA ET AL. Durability of antegrade synthetic aortomesenteric bypass for chronic mesenterio ischemia. J Vasc Surg 2002; 35: 1078-84.

10. Park WM, Cherry KJ Jr, Chua HK, Ciark RC, Jenkins G, HARMSEN WS ET AL. Cument results of open revasculanization for chronic mesenteric ischemia: a standard for comparison. J Vasc Surg 2002; 35: 853-9.

11. Furrer J, Gruntzig A, Kugelmeier J et al. Treatment of abdominal angina with percutaneous dilatation of an arteria mesenterica superior stenosis. Cardiovasc Intervt Radiol 1980; 3: 43-4.

12. van Wanroij JL, van Petersen AS, Huisman AB, Mensink PB, GerRits DG, Kolkman JJ et al. Endovascular treatment of chronic splanchnic syndrome. Eur J Vasc Endovasc Surg 2004; 28: 193-200.

13. AbuRahma AF, Stone PA, Bates MC, Weich CA. Angioplasty/Stenting of the superior mesenteric artery and celiac trunk: early and late outcomes. J Endovasc Ther 2003; 10: 1046-53. 